\title{
ROLLT IN AFRIKA DIE NATIONALISIERUNGSWELLE?
}

\author{
Von Wolfgang Heidelberg
}

In letzter Zeit konnte man insbesondere bei der Lektüre deutscher Zeitungsberichte über Afrika den Eindruck gewinnen, als häuften sich in den Staaten dieses Kontinents Verstaatlichungen oder Maßnahmen mit ähnlicher Folgewirkung. Diese Meldungen waren oftmals geeignet, beim Leser die Schlußfolgerung zu wecken, als handle es sich hierbei un ein in allen afrikanischen Staaten zu beobachtendes Phänomen, da häufig das Wort Afrika synonym für einzelne Staaten dieses Kontinents gebraucht wurde. So meldete DIE WELT vom 1. 6. 1970, daß staatliche Eingriffe in Afrika zunehmen, während das Handelsblatt vom 29./30. 5. 1970 sogar feststellte, in Afrika rolle die Nationalisierungswelle. Bedenkt man, daß gerade die Angst vor Verstaatlichungen einen der Haupthinderungsgründe für die Anlage privaten Kapitals darstellt und daß Nationalisierungen wie kaum eine andere Maßnahme geeignet sind, das Investitionsklima eines Landes nachhaltig zu verschlechtern, so kann man ermessen, daß durch unrichtige oder leichtfertig pauschalierende Darstellungen diejenigen afrikanischen Staaten geschädigt werden, die sich solcher Maßnahmen bisher enthalten haben. Geht man davon aus, daß derzeit in Afrika 42 unabhängige Staaten existieren, so haben hiervon maximal ein Drittel bisher zu irgendeinem Zeitpunkt nach Erlangung ihrer Unabhängigkeit zu Verstaatlichungsmaßnahmen gegriffen oder diesen ähnliche Eingriffe vorgenommen. Teilweise liegen jedoch diese Verstaatlichungen schon einige Jahre zurück, in vielen Fällen handelt es sich zudem um die Bereinigung des Verhältnisses zur ehemaligen Kolonialmacht, deren Gesellschaften auch nach der Erlangung der Unabhängigkeit der afrikanischen Staaten häufig ihren dominierenden Einfluß behalten hatten.

In jüngster Zeit sind Verstaatlichungen in einigen afrikanischen Staaten vorgekommen, doch sind es insbesondere die Ereignisse in Uganda und Ghana, die viel Staub aufgewirbelt haben und die man als Beispiele dafür ansehen darf, wie man eine solche Maßnahme am besten nicht durchführen sollte, selbst wenn es sich hinterher herausstellt, daß die Rechte und Interessen der Betroffenen gebührend berücksichtigt wurden.

In Uganda kündigte Präsident Obote in einer Rede zum Tag der Arbeit am 1. Mai dieses Jahres die Übernahme der Außenhandelsbetriebe und der wichtigsten gewerblichen Unternehmen sowie der Banken an. Betroffen von der Ankündigung waren 84 Unternehmen aus Industrie, Transport und Verkehr und dem Versicherungssektor, besonders aber auch die Banken, denen noch in der zweiten Jahreshälfte 1969 zugesichert worden war, daß Verstaatlichungen in den nächsten fünf Jahren nicht zu erwarten seien und die sich im Vertrauen auf diese Zusage mit Einlagen von je 20 Mill. Shs in Uganda inkorporieren ließen.

Das Gesetz, durch das die ugandische Regierung festlegte, daß sie 60 Prozent des Kapitals der betroffenen Firmen zu übernehmen gedenke - The Companies (Government and Public Bodies Participation) Act, 1970 - erging bereits am 5. Mai 1970, also nur vier Tage nach der Rede des Präsidenten. Wenn man einerseits einer verstan.tlichenden Regierung zugestehen muß, daß sie schnell vorgehen muß, um zu verhindern, daß die Betroffenen in letzter Minute noch große Vermögenswerte ihrem Zugriff entziehen, so läßt sich andererseits nicht leugnen, daß die ugandische Regierung reichlich überstürzt handelte. Für diese Vermutung 
spricht der extrem kurze Zeitraum zwischen Präsidentenrede und Verabschiedung des Gesetzes; denn gerade in Uganda liegen üblicherweise zwischen der Veröffentlichung einer Gesetzesvorlage (Bill) und ihrer Verabschiedung durch das Parlament als Gesetz (Act) Zeiträume von mehreren Wochen bzw. Monaten, in denen ausführlich das Für und Wider des Entwurfs erörtert werden kann. Wenn eine solche Erörterung wegen des oben erwähnten Überraschungseffektes nicht in Frage kam, so wäre doch etwas weniger Eile vorteilhaft gewesen. So hätte man wahrscheinlich vermeiden können, die Liste der Firmen, die von der Maßnahme betroffen und im Gesetzesanhang aufgeführt sind, nur deshalb zu ändern, weil sie noch Unternehmen enthielt, die bereits $\mathrm{zu} 100$ Prozent in ugandischem Staatsbesitz standen. Man hätte auch mehr Zeit gehabt, Art. 13 der Verfassung, der eine Enteignung nur bei Vorliegen bestimmter Voraussetzungen zuläßt, abzuändern; so mußte diese Verfassungsbestimmung ebenfalls in großer Eile und mit rückwirkender $\mathrm{Kraft}$ geändert werden. Während bisher im Enteignungs- und auch im Verstaatlichungsfall - das Gesetz selbst unterscheidet nicht zwischen beiden Alternativen, sondern legt generell fest, daß kein Eigentum zwangsweise entzogen werden darf ("no property . . . shall be compulsorily taken possession of, . . .) - prompte und angemessene Entschädigung zu zahlen war, entfällt das prompt im neuen Text ganz und das angemessen wurde durch das schwächere vernünftig (reasonable) ersetzt. Úberhaupt nicht bedacht hat man jedoch, daß sich auch das Investitionsgesetz - The Foreign Investments (Protection) Act, 1964 - in der Frage einer Enteignung auf die Verfassung bezieht und ausdrücklich feststellt, daß Enteignungen nur in Übereinstimmung mit der Verfassung erfolgen dürfen, wobei es zusätzlich die Regierung verpflichtet, eine Entschädigung spätestens binnen sechs Monaten zu zahlen. Unabhängig von der Frage, ob von den Verstaatlichungsmaßnahmen Firmen betroffen sind, die gemäß dem Investitionsförderungsgesetz den sog. "approved status" erhalten haben und die nach der derzeitigen Rechtslage im unklaren darüber sein müssen, wann sie entschädigt werden, wäre in jedem Fall ein klärendes Wort angebracht, wieweit die kurze Frist des Investitionsgesetzes in Zukunft noch Anwendung finden soll. Das Gesetz vom 5. Mai 1970 jedenfalls sieht vor, daß Entschädigungen aus den zukünftigen Gewinnen der übernommenen Firmen gezahlt werden sollen, und zwar verteilt über einen Zeitraum von höchstens 15 Jahren. Diese Regelung läßt viele Fragen offen, die in Ausführungsbestimmungen zum Gesetz oder in den mit den betroffenen Firmen zu schließenden Entschädigungsvereinbarungen zu klären sind. $\mathrm{Zu}$ diesen Fragen gehören die nach der Berücksichtigung des jährlichen Währungsverfalls oder nach der Verzinsung der jährlichen Abschlagzahlungen. Am wichtigsten jedoch dürfte eine Klärung des Problems sein, was geschehen soll, wenn Gewinne nicht oder nicht in der Höhe erzielt werden, daß die zugesagte Entschädigung gezahlt werden kann. Es dürfte für die zukünftige Wirtschaftsentwicklung Ugandas nicht unwesentlich darauf ankommen, bei der Beseitigung zahlreicher Zweifel über die effektive Bedeutung und Tragweite der neuen Bestimmungen durch klare und eindeutige Antworten und Stellungnahmen behilflich zu sein. Uganda ist bemüht, den Rückstand in seiner wirtschaftlichen, insbesondere industriellen, Entwicklung gegenüber Kenya zu verringern. Im Vertrag über die Gründung der Ostafrikanischen Gemeinschaft sind daher besondere Bestimmungen enthalten, die verhindern sollen, daß Uganda (und auch Tansania) in ihrer Entwicklung noch weiter hinter Kenya zurückbleiben. Diese Bestimmungen würden jedoch ihren Zweck nicht erreichen können, wenn gleichzeitig durch die interne Rechtslage die Sicherheit privater Investitionen nicht 
mehr gewährleistet wird; die ugandische Regierung hat bisher immer erklärt, daß sie die Anlage von Privatinvestitionen im Lande für erforderlich und wünschenswert halte; diese Ansicht hat sie bisher nicht offiziell widerrufen.

Waren es in Uganda vorwiegend innenpolitische Schwierigkeiten, die die Regierung veranlaßten, zu Verstaatlichungsmaßnahmen zu greifen, um dadurch von diesen Schwierigkeiten abzulenken, so liegen die Dinge in Ghana anders. Hier scheint eine einflußreiche Gruppe ghanaischer Geschäftsleute Druck auf die Regierung auszuüben, um zu erreichen, daß lästige ausländische Konkurrenten aus dem Markt gedrängt und die freiwerdenden Plätze von Einheimischen übernommen werden.

Die ghanaischen Maßnahmen, die man kaum als Verstaatlichungen oder Enteignungen anzusehen haben wird, sondern als Bemühungen zur Afrikanisierung oder Ghanaisierung der Wirtschaft des Landes, wobei sie allerdings für den Betroffenen die gleiche Wirkung zeigen wie Verstaatlichungen, wurden im Dezember 1968 erstmals in Form des Ghanaian Enterprises Decree getroffen. Dieses Dekret - ein formelles Gesetz konnte damals nicht ergehen, da noch das Militär an der Macht und das Parlament aufgelöst war - reservierte rückwirkend vom 1. Juli 1968 an alle Einzelhandelsbetriebe bis zu einem Jahresumsatz von 500000 Cedi, Großhandelsbetriebe bis zu 1 Million Jahresumsatz sowie einzelne Gewerbebetriebe der ausschließlichen Betätigung durch Ghanaer. Eine Reihe von Übergangsbestimmungen sollte verhindern, daß den betroffenen ausländischen Firmen zu große Schwierigkeiten erwachsen, und ihnen insbesondere die Möglichkeit einräumen, sich auf eine andere Betätigung umzustellen

Im Juli 1970 folgte als weitere Maßnahme, dieses Mal in der Form eines Gesetzes, der Ghanaian Enterprises (Promotion) Act, von dem sich viele eine Milderung der 1968 ergangenen Bestimmungen versprachen. Diese Hoffnungen wurden enttäuscht, denn das neue Gesetz fiel eher noch härter aus. So müssen z. B. am 1. August dieses Jahres alle Groß- und Einzelhandelsbetriebe, deren Kapital nicht vollständig von Ghanaern gehalten wird, schließen; ausgenommen von der Schlie. ßung sind lediglich Unternehmen, die im gesetzlich festgelegten Referenzjahr 1967/68 einen Umsatz von mindestens 500000 Cedi versteuert haben. Auf den ersten Blick schien es sich hier um eine Milderung der Bestimmungen von 1968 zu handeln, da nicht mehr vom Großhandelsumsatz von 1 Million Cedi die Rede war. Eine zusätzliche Vorschrift legt jedoch fest, daß Betriebe mit Umsätzen von mehr als 500000 Cedi nur mit einer Lizenz des zuständigen Ministers, nicht also automatisch bei Nachweis der Mindestumsatzhöhe, weiterarbeiten dürfen. Unabhängig von der Höhe des Umsatzes müssen vom 30. Juni des kommenden Jahres an u. a. Transportunternehmen, Bäckereien, Druckereien, Schönheitssalons, Werbefirmen usw. vollständig in ghanaische Hände übergegangen sein, wenn sie sich noch kommerziell betätigen wollen. Noch in diesem Jahr, und zwar wiederum ab 1. August, ist das Taxigewerbe, der Verkauf von Fahrzeugen zum Personentransport und die Tätigkeit des Vertreters für ausländische Firmen vollen Umfanges auf Ghanaer zu übertragen.

Ursprünglich, d. h. insbesondere bei Erlaß des Dekretes von 1968, wurde von ghanaischer Seite immer wieder betont, die neuerlassenen Tätigkeitsbeschränkungen seien weniger gegen Europäer, als vielmehr gegen nicht-ghanaische Afrikaner sowie gegen die den Handel beherrschenden Libanesen, Syrer, Griechen und Inder gerichtet. Es zeigte sich jedoch bald, daß auch europäische Firmen in gleicher Weise tangiert waren. Während die Europäer zunächst nur damit rechneten, ihre langjährigen Abnehmer und Kunden zu verlieren, wurde ihnen bald darauf klarge- 
macht, daß sie selbst aus dem Geschäftsleben oder wenigstens aus einigen Bereichen desselben verdrängt werden sollten. Das Unangenehme im Vorgehen der ghanaischen Regierung ist die Art, in der die betroffenen Firmen oder Kaufleute aus dem Lande getrieben werden. Da man die ergriffenen Maßnahmen nicht als Verstaatlichung oder Enteignung ansieht, ist auch an keiner Stelle der Bestimmungen von Entschädigungen die Rede. Man räumt vielmehr den Betroffenen lediglich die Alternative ein, vor Ablauf der jeweiligen Stichtage ihre Betriebe an Ghanaer zu übertragen oder mit Eintritt des Termins die Tätigkeit einfach einzustellen. Abgesehen von der Frage, ob es überhaupt genügend ghanaische Bürger gibt, die das erforderliche Kapital zum Kauf der Betriebe besitzen oder zu beschaffen in der Lage sind, bleibt zu klären, wie diese Veräußerung tatsächlich vor sich geht und was sie für den Veräußerer bedeutet. Es dürfte ausgeschlossen sein, daß er einen angemessenen Preis erzielen kann, denn der Erwerber hat den Vorteil des Zeitablaufs auf seiner Seite und weiß, daß der Veräußerer verkaufen muß und wahrscheinlich auch wird, weil diese Lösung immer noch vorteilhafter sein wird als eine schlichte Betriebsaufgabe. Und selbst nach einer Veräußerung hat der Verkäufer nicht eben viel gewonnen, denn der ghanaische Cedi ist nicht konvertibel und infolge der Devisenknappheit des Landes wird die Bewirtschaftung sehr streng gehandhabt.

Unerfreulich an den ghanaischen Bestimmungen ist aber in ganz besonderem Maße, daß sie teilweise unklar sind und daß sich die ghanaische Regierung trotz intensiver Bemühungen der Betroffenen und ihrer diplomatischen Vertretungen, Klarheit $\mathrm{zu}$ erlangen, nicht bereit findet, aufgetretene $\mathrm{Zweifel} \mathrm{zu}$ beseitigen. Im Gegenteil, die Hoffnung, durch Ausführungsbestimmungen zum Gesetz werde ein Teil dieser Zweifelsfragen geklärt werden, ist durch die Regierungserklärung zunichte gemacht worden, solche Bestimmungen würden nicht ergehen. So sind insbesondere die europäischen Firmen und Kaufleute im unklaren darüber, wer als "Vertreter einer überseeischen Firma" (overseas business representation) anzusehen ist und somit die Tätigkeit einzustellen hat. Ein erster Gesetzentwurf, der inzwischen zurückgezogen wurde, war in diesem Punkt klar und eindeutig und nahm diejenigen Vertreter vom Betätigungsverbot aus, die einen komplizierten technischen Service nach Verkauf ihrer Ware bieten. Das Gesetz in seiner jetzigen Endfassung definiert den Vertreterbegriff als „.. . the carrying on of business in Ghana by any person as sole agent for the sale or promotion of the sale of a product or group of products of a principal not resident in Ghana, in consideration for remuneration or other financial reward from the principal". Bedeutet die Verwendung des Begriffs sole agent, daß nur Alleinvertreter betroffen sind, und genügt es demzufolge, die Eigenschaft des Alleinvertreters aufzugeben, indem man einer anderen Person oder Firma die Vertretung gegebenenfalls überträgt, damit man weiterarbeiten kann? Oder wird es die ghanaische Regierung gegebenenfalls darauf ankommen lassen, daß ein bestimmtes Produkt im Lande nicht mehr verkauft wird, weil sich der ausländische Hersteller weigert, seine Ware über mehr als einen Vertreter absetzen zu lassen, wozu ihn die ghanaische Regierung schließlich nicht zwingen kann, da er ihrer Hoheitsgewalt entzogen ist?

Es versteht sich von selbst, daß das Ghanaisierungsgesetz zahlreiche Vorschriften enthält, die der Regierung die Kontrolle darüber einräumen, daß die Bestimmungen auch eingehalten werden. So sind Verschmelzungen genehmigungspflichtig, weil man vermeiden will, daß durch den Zusammenschluß verschiedener Firmen die im Gesetz als Grenzen gesetzten Umsätze erreicht werden, damit die Tätigkeit 
fortgesetzt werden kann. Besondere Inspektoren überprüfen die Befolgung des Gesetzes, ihnen ist (richtige) Auskunft zu erteilen und Einsicht in die Unterlagen zu gewähren. Daß das Gesetz aber jedem Angestellten (any person) der betroffenen Firma diese Pflichten auferlegt und bei Nichteinhaltung strenge Strafen (bis zu zwei Jahren Gefängnis und 2000 Cedi Geldstrafe) androht, anstatt nur auf die in der Regel hierfür zuständigen leitenden Angestellten zurückzugreifen, könnte zu kaum zu rechtfertigenden Eingriffen in Betriebsführung und -geheimnisse führen.

Die Hoffnung der ausländischen Firmen richtet sich für die Zukunft daher am stärksten auf die Bestimmung des Gesetzes, die es dem zuständigen Minister nach Zustimmung des Kabinetts erlaubt, Personen oder Betriebe von der Einhaltung aller oder eines Teils der Bestimmungen des Gesetzes zu befreien. Zu groß dürfte diese Hoffnung aber nicht sein; denn es handelt sich um eine reine Kann-Vorschrift, außerdem steht die Regierung unter dem nicht zu unterschätzenden Druck ghanaischer Geschäftsleute, die die Hoffnung haben, stärkeren Einfluß auf die Wirtschaft des Landes zu gewinnen.

Die beiden soeben geschilderten Beispiele Ugandas und Ghanas dürften klar gezeigt haben, daß es im Falle von Verstaatlichungen oder ähnlichen Eingriffen im Interesse der jeweils handelnden Regierung selbst liegt, klare und eindeutige Entscheidungen zu treffen, die keinen Anlaß zu Spekulationen über ihre Tragweite und Bedeutung geben. Verstaatlichungen werden sicherlich niemals von den Betroffenen freudig begrüßt werden; andererseits kann kein Staat gehindert werden, sie durchzuführen, wenn dies im Interesse des Gesamtwohls erforderlich ist. Es ist jedoch in jedem Falle verfehlt, aufgrund der Ereignisse in Ghana und Uganda und einiger weiterer Beispiele von einer Verstaatlichungswelle in Afrika zu sprechen; man tut mit dieser Pauschalbehauptung mindestens 25 Staaten des Kontinents, u. a. nahezu allen mit der EWG assoziierten Staaten unrecht. Vertraut machen sollte man sich jedoch mit dem Gedanken, daß die Afrikanisierungsbestrebungen sich erst in ihrem Anfangsstadium befinden; die afrikanischen Regierungen wollen mehr eigene Staatsangehörige in den leitenden Positionen von Wirtschaft und Verwaltung sehen. Dieses Anliegen ist um so legitimer, je mehr fachlich qualifizierte Afrikaner zur Verfügung stehen. Die ausländischen Firmen sollten sich daher bemühen, dieses Fachwissen den Afrikanern zu vermitteln; dann wird sich der Austausch des europäischen Personals durch einheimische Kräfte reibungslos und zum Nutzen aller Beteiligten vollziehen. 